\title{
molecules
}

ISSN 1420-3049

www.mdpi.com/journal/molecules

Article

\section{Synthesis and in Vitro Antimicrobial Evaluation of New N-Heterocyclic Diquaternary Pyridinium Compounds}

\section{Bianca Furdui ${ }^{1}$, Georgiana Parfene ${ }^{2}$, Ioana Otilia Ghinea ${ }^{2}$, Rodica Mihaela Dinica ${ }^{1, *}$, Gabriela Bahrim ${ }^{2}$ and Martine Demeunynck ${ }^{3,4}$}

1 Department of Chemistry, Physics and Environment, Faculty of Science and Environment, "Dunarea de Jos" University of Galati, 111 Domneasca Street, 800201 Galati, Romania; E-Mail: bfurdui@ugal.ro

2 Department of Food Science, Food Engineering and Applied Biotechnology, Faculty of Food Science and Engineering, "Dunarea de Jos" University of Galati, 111 Domneasca Street, 800201 Galati, Romania; E-Mails: Georgiana.parfene@ugal.ro (G.P.); ioana.ghinea@ugal.ro (I.O.G.); bahrim.gabriela@ugal.ro (G.B.)

3 Centre National de la Recherche Scientifique (CNRS), UMR 5063, Department of Molecular Pharmacochemistry, F-38041 Grenoble, France; E-Mail: martine.demeunynck@ujf-grenoble.fr

4 Department of Molecular Pharmacochemistry, University of Grenoble-Alpes, F-38041 Grenoble, France

* Author to whom correspondence should be addressed; E-Mail: rodinica@ugal.ro; Tel.: +40-0336-130-251; Fax: +40-0336-130-285.

Received: 11 May 2014; in revised form: 25 July 2014 / Accepted: 28 July 2014 / Published: 5 August 2014

\begin{abstract}
A series of bis-pyridinium quaternary ammonium salts (bis-PyQAs) with different aryl and heteroaryl moieties were synthesized and their antimicrobial activity investigated. The inhibition effect of the compounds was evaluated against bacteria, molds and yeasts; the activities were expressed as the minimum inhibitory concentrations (MIC). The relationships between the structure descriptors (logP, polarizability, polar surface area (2D), van der Waals area (3D)) and the biological activity of the tested bis-PyQAs are discussed.
\end{abstract}

Keywords: pyridinium quaternary salts; antimicrobial activity; 4-[2-(pyridin-4yl)ethyl]pyridine, 4,4'-bipyridine 


\section{Introduction}

Quaternary ammonium salts and their variously substituted derivatives are important biological agents and have found applications in different fields [1-4]. In particular, they are used as excellent antimicrobial agents against both Gram-positive and Gram-negative bacteria [5-10]. Some of these compounds have antifungal, hemolytic and cytotoxic properties [11]. Quaternary ammonium salts are also widely used for paint, water treatment, textile and sanitizing food preparation areas, because of their relatively low toxicity and broad antimicrobial activity $[12,13]$. Pyridinium salts constitute a versatile class of compounds used as phase transfer catalysts [14], photosensitizing dyes in polymerization reactions [15] and cationic surfactants [16]. Moreover, pyridinium species have played a crucial role in the development of cycloaddition reactions in heterocycle synthesis and have been extensively used in organic synthesis [17]. Selected salts are also found as ionic liquids [18], and viologens (4,4'-bipyridinium salts) demonstrate electrochromic properties [19].

In recent years, a significant part of the research in heterocyclic chemistry has been devoted to the synthesis and study of variously substituted pyridinium salts. The general synthesis of these salts involves the reaction of pyridine derivatives with reactive halides [20,21-23]. This paper focuses on the synthesis and characterization of bis-pyridinium quaternary ammonium salts (bis-PyQAs) starting from 4,4'-bipyridine or 4-[2-(pyridin-4-yl)ethyl]pyridine, as well as the evaluation of their antimicrobial activity. The antimicrobial properties were evaluated using different spoilage microorganisms, which can be found in food microbiota. The relationships between structure, molecular hydrophobicity, solubility, and the biological activity of the tested bis-PyQAs are also discussed.

\section{Results and Discussion}

\subsection{Synthesis}

All nine derivatives were prepared using the methodology shown in Scheme 1. Thus, the synthesis of the diquaternary bispyridinium salts $4 \mathbf{a}-\mathbf{e}$ has been carried out through the alkylation of 4-[2-(pyridin-4-yl)ethyl]pyridine 1 with reactive halogenated reagents in anhydrous acetonitrile, which seems to be the most convenient method reported in the literature [21]. The diquaternary bispyridinium salts 5a-d were prepared using similar conventional methodology [22], from 4,4'-bipyridine 2 . The products were easily purified by washing with boiling solvent.

The alkylation reaction was influenced by the type of substituent present on the alkylating reagent, and it appeared that the reactivity of the -halo ketones followed the order depicted in Scheme 2. The lower reactivity of $\mathbf{3 e}$ is likely due to the lower reactivity of the chloro-alkyl reagent.

The structures of all compounds were confirmed by IR, ${ }^{1} \mathrm{H}-\mathrm{NMR}, \mathrm{COSY},{ }^{13} \mathrm{C}-\mathrm{NMR}$, MS and elemental analysis, whereas their purity was confirmed by HPLC.

IR spectra of all compounds have been investigated in the frequency range 4,000-650 $\mathrm{cm}^{-1}$ and the observed absorption bands support the proposed structures: $3,375-3,001 \mathrm{~cm}^{-1}$ (CH arom), $2,988-2,820 \mathrm{~cm}^{-1}$ (CH aliph), 1,747-1,667 $\mathrm{cm}^{-1}(\mathrm{C}=\mathrm{O}), 1,645-1,637 \mathrm{~cm}^{-1}(\mathrm{C}=\mathrm{N}), 1,525-1,519$ and $1,342-1,338 \mathrm{~cm}^{-1}\left(\mathrm{NO}_{2}\right), 1,245-1,241 \mathrm{~cm}^{-1}, 1,201-1,188 \mathrm{~cm}^{-1}$ (C-O-C) and $\sim 996-923 \mathrm{~cm}^{-1}$ (C-C aliph). 
Scheme 1. Synthesis of diquaternary salts of bispyridinium.
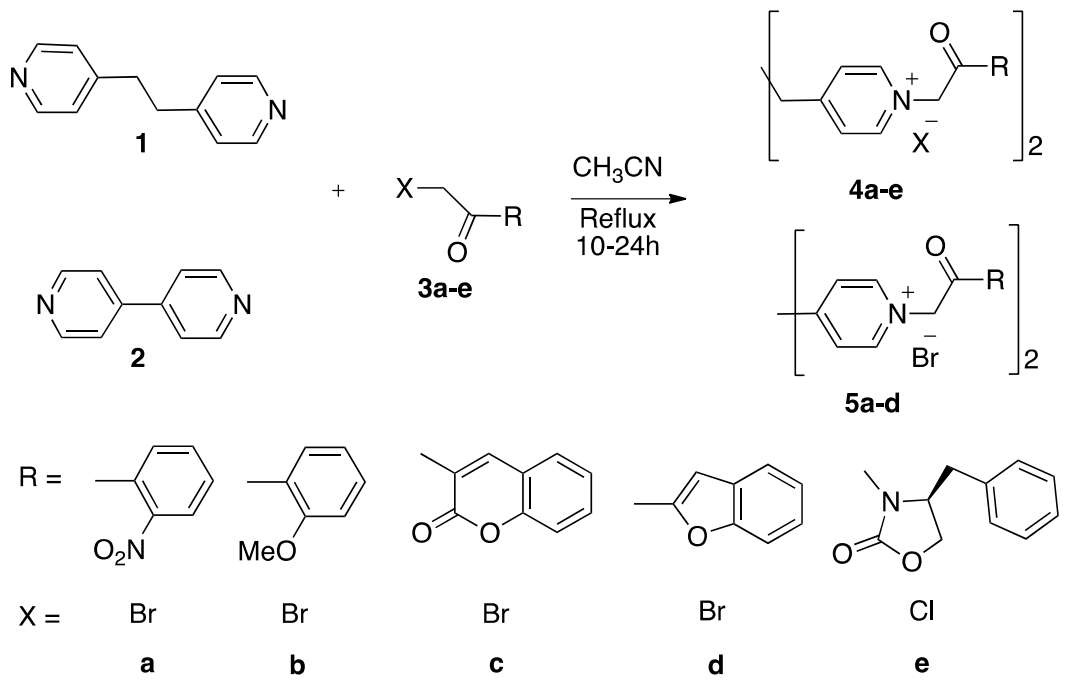

Scheme 2. The reactivity of $\alpha$-halo ketones in nucleophilic substitution.

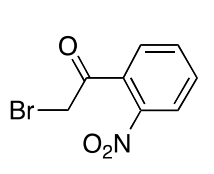

$3 \mathbf{a}$

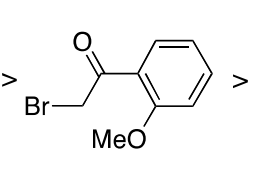

3b<smiles>O=C(CBr)c1cc2ccccc2oc1=O</smiles>

$3 c$

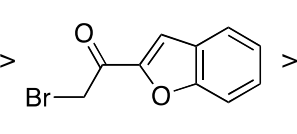

$3 d$<smiles>O=C(CCl)N1C(=O)OCC1Cc1ccccc1</smiles>

$3 e$

The structures of salts were further confirmed by ${ }^{1} \mathrm{H}-\mathrm{NMR}$ and COSY analysis. All of these compounds showed multiple peaks in the region of 9.65-7.20 ppm that were assigned to aromatic protons. Additionally, the observed peaks at $6.47-6.14 \mathrm{ppm}$ corresponded to the $\mathrm{CH}_{2}$ units linked to the cationic nitrogen, whereas the peaks centered at 3.52-3.47 ppm were attributed to the ethylene bridge protons of Compounds $\mathbf{4 a - e}$. For salt $\mathbf{4 e}$, each proton of the benzyl moiety, and those from the oxazolidinone ring appeared as coupled multiplets due to its specific configuration.

${ }^{13} \mathrm{C}$-NMR spectra also confirmed the structures. Thus, for all compounds, the signals of the quaternary carbon of carbonyl groups were found at 192.83-165.74 ppm, and the signals of the lactone carbonyl present in 4c, 4e and 5c were observed at 160.53-158.73 ppm. At higher fields, the signals at 69.98-61.04 ppm represented the $\mathrm{CH}_{2}$ groups close to the pyridinium nitrogens, in 5a-5e, whereas the signals at $\sim 33$ ppm were attributed to the ethylenic carbons of salts $4 \mathbf{a}-\mathbf{4 e}$. Due to the ionic structure of the synthesized compounds, their electrospray mass spectra (ESI-MS) gave major peaks corresponding to the organic ions $\left[\mathrm{M}^{2+}-\mathrm{H}^{+}\right]$, with masses between 453 and 619 .

\subsection{Antimicrobial Activity}

We have previously investigated the antimicrobial properties of some diquaternary ammonium salts and found that they exerted a significant antimicrobial action against bacteria, yeasts and molds [22,24,25]. In the present study, the compounds were tested against nine different microorganisms: Gram-positive bacteria (Bacillus subtilis, Bacillus cereus, Sarcina lutea), yeasts (Rhodotorula glutinis, Candida utilis, Saccharomyces cerevisiae) and molds (Aspergillus niger, Geotrichum candidum, Penicillium roqueforti). The qualitative results obtained by disk diffusion assay are presented in Table 1. 
Table 1. Diameters expressed in mm of growth inhibition zone of the bis-pyridinium quaternary ammonium salts (bis-PyQAs) $\mathbf{4 a - d}$ and $\mathbf{5 a}-\mathbf{d}$. Values are presented as the mean \pm SEM. The diameter of the test paper discs is $19 \mathrm{~mm}$.

\begin{tabular}{|c|c|c|c|c|c|c|c|c|c|}
\hline MO & $4 \mathbf{a}$ & $4 b$ & $4 c$ & $4 d$ & $5 a$ & $5 b$ & $5 c$ & $5 d$ & $\mathrm{H}_{2} \mathrm{O}$ \\
\hline B. subtilis & $35.00 \pm 0.57$ & $30.33 \pm 0.33$ & $19.16 \pm 0.16$ & $40.83 \pm 0.44$ & $41.33 \pm 0.33$ & $45.00 \pm 0.57$ & $19.83 \pm 0.44$ & $25.33 \pm 0.33$ & 0 \\
\hline B cereus & $41.66 \pm 0.33$ & $43.00 \pm 0.57$ & $19.33 \pm 0.33$ & $37.33 \pm 0.33$ & $35.50 \pm 0.28$ & $50.50 \pm 0.28$ & $19.50 \pm 0.28$ & $21.16 \pm 0.16$ & 0 \\
\hline S. lutea & $22.33 \pm 0.33$ & $19.50 \pm 0.28$ & $41.00 \pm 0.57$ & $19.50 \pm 0.28$ & $46.00 \pm 0.57$ & $45.33 \pm 0.33$ & $21.50 \pm 0.28$ & $41.50 \pm 0.28$ & 0 \\
\hline S. cerevisiae & $22.00 \pm 0.57$ & $22.66 \pm 0.66$ & $19.66 \pm 0.66$ & $19.16 \pm 0.16$ & $19.50 \pm 0.28$ & $23.16 \pm 0.16$ & $19.33 \pm 0.16$ & $19.33 \pm 0.33$ & 0 \\
\hline C. utilis & $22.66 \pm 0.66$ & $22.50 \pm 0.28$ & $19.33 \pm 0.33$ & $19.33 \pm 0.33$ & $19.16 \pm 0.16$ & $22.66 \pm 0.66$ & $19.33 \pm 0.33$ & $19.16 \pm 0.16$ & 0 \\
\hline$R$. glutinis & $40.33 \pm 0.33$ & $35.33 \pm 0.33$ & $19.50 \pm 0.28$ & $19.83 \pm 0.44$ & $19.66 \pm 0.33$ & $53.50 \pm 0.28$ & $19.50 \pm 0.28$ & $19.50 \pm 0.50$ & 0 \\
\hline A. niger & $19.66 \pm 0.66$ & $19.33 \pm 0.33$ & $19.83 \pm 0.44$ & $19.33 \pm 0.33$ & $19.33 \pm 0.33$ & $32.33 \pm 0.33$ & $19.33 \pm 0.33$ & $19.16 \pm 0.16$ & 0 \\
\hline P. roqueforti & $19.50 \pm 0.28$ & $19.16 \pm 0.16$ & $19.16 \pm 0.16$ & $19.16 \pm 0.16$ & $19.16 \pm 0.16$ & $19.16 \pm 0.16$ & $19.16 \pm 0.16$ & $19.50 \pm 0.28$ & 0 \\
\hline G. candidum & $20.00 \pm 0.57$ & $21.66 \pm 0.66$ & $19.33 \pm 0.33$ & $19.33 \pm 0.16$ & $19.33 \pm 0.33$ & $33.16 \pm 0.16$ & $19.16 \pm 0.16$ & $19.33 \pm 0.33$ & 0 \\
\hline
\end{tabular}


In this test, a compound was considered as active if the difference between the growth inhibition zones of this product and the blank was at least $2 \mathrm{~mm}$. The following classification of the chemical compounds activity was proposed, depending on the dimensions of the inhibition zones (Diz): with low inhibitory effect, Diz $\leq 20 \mathrm{~mm}$; with medium inhibitory effect, Diz $=20-50 \mathrm{~mm}$; and with strong inhibitory effect, Diz $\geq 50 \mathrm{~mm}$ [26]. Previous studies showed that the antimicrobial activity of pyridinium quaternary salts is based on their action on the cell wall, and the factors that control the antimicrobial activity include the molecular hydrophobicity [27], adsorbability or electron density of the nitrogen atom $[28,29]$. When used in water treatment, the high solubility of the antimicrobial agent is mandatory. Indeed, due to the presence of two positive charges on the structures, all compounds displayed good solubility in water. In an effort to establish structure-activity relationships, several parameters were determined. The relative lipophilicity $\left(\mathrm{R}_{\mathrm{M}}\right)$ of the tested molecules was measured using thin-layer partition chromatography. Higher $\mathrm{R}_{M}$ values indicate greater lipophilicity. Other parameters, including 2D descriptors representing physical properties $(\log \mathrm{P}), 3 \mathrm{D}$ descriptors (van der Waals surface area) and quantum chemical parameters (polarizability) were calculated using available on-line chemical software [30]. The data are collected in Table 2. Concerning hydrophobicity, $\mathbf{4 b}$ and 5b displayed the highest molecular hydrophobicity with $\mathrm{R}_{\mathrm{M}}$ values of 2.32 and 2.14 . The $\log \mathrm{P}$ parameter was in agreement with $\mathrm{R}_{\mathrm{M}}$, as exemplified by the low value calculated for $\mathbf{5 b}$. It is worth noting that the bispyridinium derivatives 5a-d showed lower $\log \mathrm{P}$ and van der Waals surface area values than their analogues containing the ethyl linkage between the two pyridinium rings $(\mathbf{4 a}-\mathbf{d})$. The variation of the polar surface area appeared strongly linked to the nature of the R group (see Scheme 1).

Table 2. Physicochemical properties of synthesized salts. LogP, polar and van der Waals surface areas and polarizability were calculated using MarvingSketch 6.0.2. $\mathrm{R}_{\mathrm{M}}$ values are presented as the mean $\pm \mathrm{SEM}$.

\begin{tabular}{|c|c|c|c|c|c|c|}
\hline Compound & $\mathbf{m p}\left({ }^{\circ} \mathrm{C}\right)$ & $\log P$ & $\begin{array}{c}\text { Polar surface area } \\
\left(\AA^{2}\right)\end{array}$ & $\begin{array}{c}\text { van der Waals surface area } \\
\qquad\left(\AA^{2}\right)\end{array}$ & Polarizability & $\begin{array}{c}\text { Hydrophobicity } \\
\left(\mathbf{R}_{M}\right) \\
\end{array}$ \\
\hline $4 a$ & $>250$ & -3.4 & 133.5 & 711.4 & 52.5 & $2.091 \pm 0.027$ \\
\hline $4 b$ & $241-243$ & -3.6 & 60.3 & 730.7 & 53.6 & $2.325 \pm 0.014$ \\
\hline $4 c$ & $>300$ & -3.0 & 94.5 & 732.4 & 59.5 & $2.122 \pm 0.047$ \\
\hline $4 d$ & $>300$ & -3.2 & 68.2 & 707.4 & 57.6 & $1.938 \pm 0.023$ \\
\hline $5 \mathbf{b}$ & $252-253$ & -4.5 & 60.3 & 667.1 & 51.6 & $2.142 \pm 0.024$ \\
\hline $5 c$ & $>300$ & -3.9 & 94.5 & 671.0 & 57.3 & $1.917 \pm 0.049$ \\
\hline $5 d$ & $301-302$ & -4.1 & 68.2 & 646.0 & 55.4 & $2.092 \pm 0.023$ \\
\hline
\end{tabular}

If we analyze these data, several general comments can be made. Significantly, all of the salts showed antimicrobial activity with a medium or strong inhibitory effect, against at least one of the tested organisms. Qualitative results revealed that the tested compounds were more effective against bacteria than against yeasts or molds. The three bacterial strains exhibited sensitivity to several molecules, 5a and $\mathbf{5 b}$ being active against these strains. The most sensitive bacterial strain was Bacillus cereus, its growth being strongly or moderately inhibited by six of the tested salts. However, Compounds $\mathbf{4 a - c}$ and $\mathbf{5 d}$ proved more active on one strain (Bacillus cereus for $\mathbf{4 a}$ and $\mathbf{4 b}$ and Sarcina lutea for $\mathbf{4} \mathbf{c}$ and $\mathbf{5 d}$ ). If we now tentatively correlate structure and activity, it appears that the most active salts, 5a and $\mathbf{5 b}$, present $\operatorname{low} \log \mathrm{P}(-4.3$ and -4.5 , respectively), polarizability (50.3 and 51.6, 
respectively) and van der Waals surface area (652.1 and $667.1 \AA^{2}$, respectively) compared to the other compounds. However, these two salts differ in polar surface area (133.5 vs. $\left.60.3 \AA^{2}\right)$ and $\mathrm{R}_{\mathrm{M}}$ (1.73 vs. 2.14) values. For comparison, the least active salt $\mathbf{5 c}$ has higher values of $\log \mathrm{P}(-3.9)$, and polarizability (57.3).

Considering the effect on yeasts, Rhodotorula glutinis seemed to be the most sensitive strain, three of the tested chemical compounds (4a, $\mathbf{4 b}$ and $\mathbf{5 b}$ ) showing strong or moderate activity against it. Again, 5b emerged as the most active salt. The three salts have high $\mathrm{R}_{\mathrm{M}}$ values $(>2)$, $\operatorname{low} \log \mathrm{P}(-3.4$ to -4.5) and low polarizability (51.6-53.6).

Finally, the bis-PyQAs were marginally active against Penicillium roqueforti, and only $\mathbf{5 b}$ salt was moderately active against Aspergillus niger. Geotrichum candidum was slightly inhibited by only three of the tested chemical compounds.

To sum up these observations, it is clear that significant differences in the inhibition of the same microorganisms by the different salts were observed. However, it seems possible to distinguish critical structural features. Compounds 5a-e derived from 4,4'-bipyridine are more active than $\mathbf{4 a}-\mathbf{e}$ derived from 4-[2-pyridin-4-yl)ethyl]pyridine. In each series, the most active molecules (4a, $\mathbf{4 b}$ and $\mathbf{5 a}, \mathbf{5 b})$ bear a phenyl ring as the R substituent. Compound $\mathbf{5 b}$, containing an electron-donating methoxy group on this phenyl ring, exhibited the highest activity against the Gram-positive bacteria and yeasts. On the other hand, Compounds $\mathbf{4 c}$ and $\mathbf{5 c}$, containing a chromene ring, showed the lowest activities.

Having these qualitative results in hand, the quantitative evaluation of the antimicrobial efficacy was made by determination of the MIC, for the most active bis-PyQAs, $\mathbf{4 b}$ and $\mathbf{5 b}$, against two bacterial strains, Bacillus cereus and Sarcina lutea. The culture growth was evaluated by measuring the increasing turbidity associated with veil formation at the culture surface for the $B$. cereus and with sediment formation for $S$. lutea. From the measured optical densities $\left(\mathrm{OD}_{620 \mathrm{~nm}}\right)$, it appeared clearly that the highest activity was observed for $\mathbf{5 b}$ with a MIC of $0.3125 \mathrm{mg} / \mathrm{mL}$ for both bacterial strains, compared to MIC values of $0.625 \mathrm{mg} / \mathrm{mL}$ for $B$. cereus and of $2.5 \mathrm{mg} / \mathrm{mL}$ for $S$. lutea for $\mathbf{4 b}$ (Figures 1 and 2).

Figure 1. In vitro antibacterial activities. Minimal inhibition concentration (MIC) value of $\mathbf{4 b}$ and $\mathbf{5 b}$ against $S$. lutea.

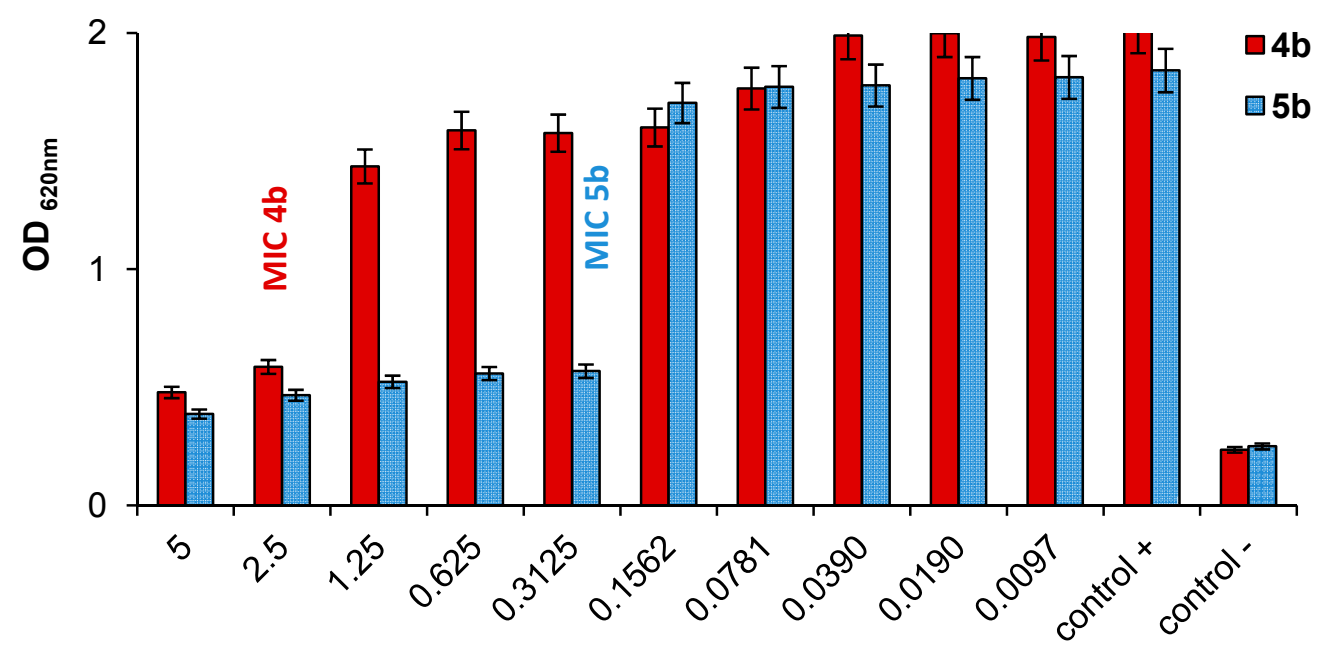

Tested serial dilutions (mg chemical compound/mL medium) 
Figure 2. In vitro antibacterial activities. Minimal inhibition concentration (MIC) value of $\mathbf{4 b}$ and $\mathbf{5 b}$ against $B$. cereus.

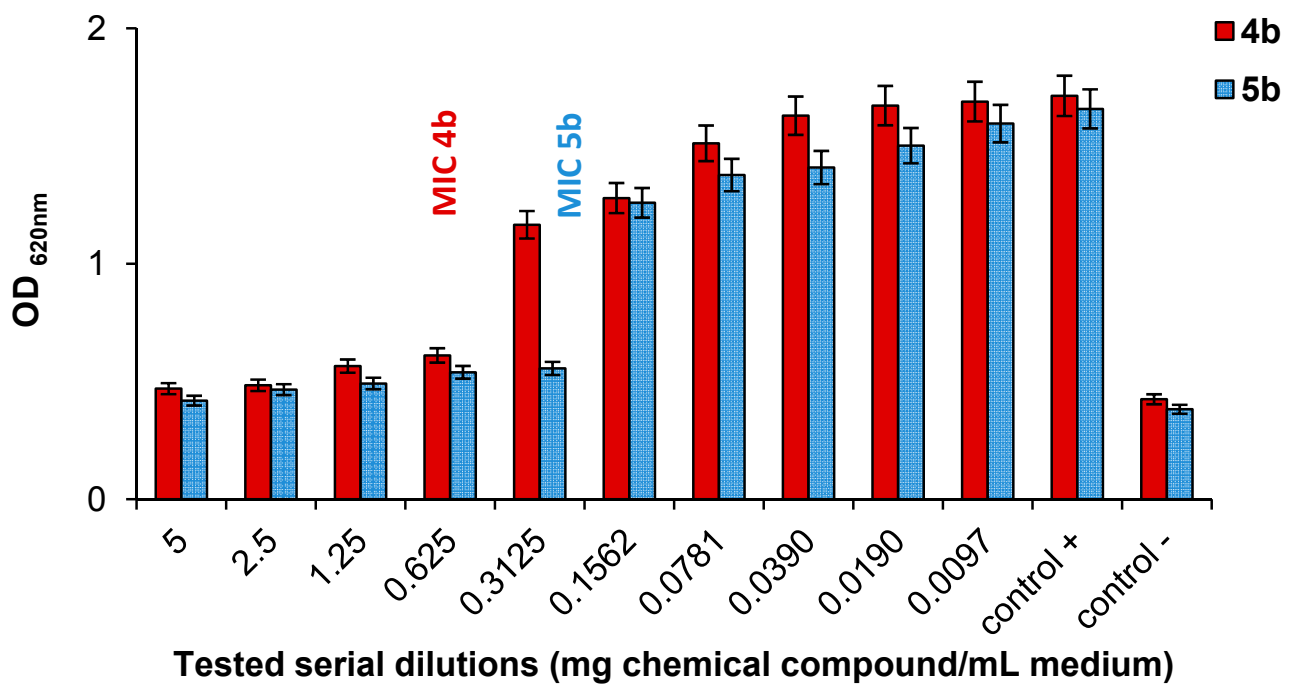

These data are fully in agreement with the qualitative observations, and Compound $\mathbf{5 b}$ emerges as a promising antibacterial agent. Due to its ionic character, this salt also displays good water solubility despite its rather high hydrophobicity. Our previous studies also showed that these types of antimicrobial agents are heat-resistant [21], which is particularly important if they are incorporated into plastics.

\section{Experimental Section}

\subsection{General}

Reagents used for synthesis were purchased from Aldrich, Fluka and Merck; organic solvents were purchased from Merck. Melting points were recorded with a Stuart SMP10 instrument. IR spectra were recorded from 4,000 to $650 \mathrm{~cm}^{-1}$ with a Perkin-Elmer Spectrum 100 instrument by ATR (Attenuated Total Reflectance) technique on a CdSe crystal. ${ }^{1} \mathrm{H}-\mathrm{NMR}, \mathrm{COSY}$ and ${ }^{13} \mathrm{C}$-NMR spectra were recorded with a Bruker 400 Ultrashield spectrometer (at $400 \mathrm{MHz}$ for ${ }^{1} \mathrm{H}-\mathrm{NMR}$ and at $100 \mathrm{MHz}$ for ${ }^{13} \mathrm{C}-\mathrm{NMR}$ ) operating at room temperature, using DMSO- $d_{6}$ as the solvent and TMS as the internal standard. Abbreviations for the data quoted are: s, singlet; $d$, doublet; $t$, triplet; q, quartet; dd, doublet of doublets; ddd, doublet of doublet of doublets; m, multiplet. The electrospray ionization (ESI) mass spectra were measured on a Thermo Scientific HPLC/MSQ Plus. Elemental analyses (C, H, N) were performed with a Fisons Instruments 1108 CHNS-O elemental analyzer. The solubility of the compounds was judged by visual observation after agitation in water for $24 \mathrm{~h}$ at $25^{\circ} \mathrm{C}$. The evaluation of minimum inhibitory concentration (MIC) was made using a Tecan Infinite 200 PRO NanoQuant Microplate reader. The microplate assay was done in a 96 -well microplate, and the absorbance in each well was read at the wavelength of $620 \mathrm{~nm}$. 


\subsection{Synthesis}

Experimental details for the synthetic procedures and analytical data and spectra of the pyridinium salts are described. The corresponding bis-pyridine (1 or 2) $(2 \mathrm{mmol})$ and halide derivatives (4.4 mmol) were refluxed in acetonitrile $(20 \mathrm{~mL})$ for 10 to $24 \mathrm{~h}$. The mixture was cooled to room temperature. The solvent was removed by filtration, and the solid thus obtained was washed with hot acetonitrile. The compounds showed high purity, as assessed by spectroscopic and elemental analyses, and were used without further purification.

1-[2-(2-Nitrophenyl)-2-oxoethyl]-4-(2-\{1-[2-(2-nitrophenyl)-2-oxoethyl]pyridin-1-ium-4-yl\}ethyl)pyridin1-ium dibromide (4a); Beige crystals; yield 93\%; $\mathrm{mp}>250{ }^{\circ} \mathrm{C}$ (decomposition). IR $\left(\mathrm{cm}^{-1}\right): 3,051$, 2,895, 2,820, 2,356, 1,715, 1,637, 1,570, 1,519, 1,473, 1,342, 1,220, 1,196, 996. ${ }^{1} \mathrm{H}-\mathrm{NMR}$ (DMSO-d ${ }_{6}$ )

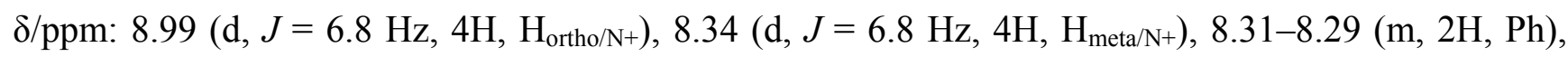
8.12-8.05 (m, 4H, Ph), 7.98-7.94 (m, 2H, Ph), $6.39\left(\mathrm{~s}, 4 \mathrm{H}, \mathrm{CH}_{2} / \mathrm{N}^{+}\right), 3.52\left(\mathrm{~s}, 4 \mathrm{H}, \mathrm{CH}_{2}-\mathrm{CH}_{2}\right) .{ }^{13} \mathrm{C}-\mathrm{NMR}$ $\left(\mathrm{DMSO}-d_{6}\right) \delta$ ppm: $192.83(\mathrm{C}=\mathrm{O}), 161.61(\mathrm{C}), 145.96(\mathrm{C}), 145.45(\mathrm{CH}), 134.68(\mathrm{CH}), 133.25(\mathrm{C})$, $131.24(\mathrm{CH}), 128.88(\mathrm{CH}), 127.84(\mathrm{CH}), 124.68(\mathrm{CH}), 66.70\left(\mathrm{CH}_{2}\right), 33.81\left(\mathrm{CH}_{2}\right) . \mathrm{MS}(\mathrm{ESI}+), m / z: 511$ $\left(\mathrm{M}^{2+}-\mathrm{H}^{+}\right)$. Anal. calcd. for $\mathrm{C}_{28} \mathrm{H}_{24} \mathrm{Br}_{2} \mathrm{~N}_{4} \mathrm{O}_{6}(\mathrm{M}=672.32 \mathrm{~g} / \mathrm{mol})$ : C, 50.02; H, 3.60; N, 8.33. Found. C, 49.92; H, 3.71; N, 8.25.

\section{1-[2-(2-Methoxyphenyl)-2-oxoethyl]-4-(2-\{1-[2-(2-methoxyphenyl)-2-oxoethyl]pyridin-1-ium-4-}

yl\}ethyl)pyridin-1-ium dibromide (4b); White crystals; yield 92\%; mp 241-243 ${ }^{\circ} \mathrm{C}$ (decomposition). IR $\left(\mathrm{cm}^{-1}\right)$ : 3,001, 2,945, 2,876, 2,358, 1,667, 1,639, 1,594, 1,520, 1,484, 1,466, 1,435, 1,337, 1,285, 1,241, 1,207, 1,188, 1,162, 1,112, 1,010, 987. ${ }^{1} \mathrm{H}-\mathrm{NMR}$ (DMSO-d 6 ) $\delta / \mathrm{ppm}: 8.95$ (d, $J=6.8 \mathrm{~Hz}, 4 \mathrm{H}$, $\left.\mathrm{H}_{\text {ortho/ } \mathrm{N}+}\right), 8.23\left(\mathrm{~d}, J=6.8 \mathrm{~Hz}, 4 \mathrm{H}, \mathrm{H}_{\text {meta/ }}+\right), 7.90(\mathrm{dd}, J=7.8,1.6 \mathrm{~Hz}, 2 \mathrm{H}, \mathrm{Ph}), 7.79-7.74(\mathrm{~m}, 2 \mathrm{H}, \mathrm{Ph})$, $7.38(\mathrm{~d}, J=8.0 \mathrm{~Hz}, 2 \mathrm{H}, \mathrm{Ph}), 7.19-7.15(\mathrm{~m}, 2 \mathrm{H}, \mathrm{Ph}), 6.22\left(\mathrm{~s}, 4 \mathrm{H}, 2 \mathrm{CH}_{2} / \mathrm{N}^{+}\right), 4.06\left(\mathrm{~s}, 6 \mathrm{H}, \mathrm{OCH}_{3}\right), 3.48$ $\left(\mathrm{s}, 4 \mathrm{H}, \mathrm{CH}_{2}-\mathrm{CH}_{2}\right) \cdot{ }^{13} \mathrm{C}-\mathrm{NMR}$ (DMSO-d $)_{6} \delta \mathrm{ppm}: 190.19(\mathrm{C}=\mathrm{O}), 160.76(\mathrm{C}), 160.08(\mathrm{CH}), 145.68$ $(\mathrm{CH}), 136.46(\mathrm{C}), 130.40(\mathrm{CH}), 127.24(\mathrm{CH}), 122.86(\mathrm{C}), 120.97(\mathrm{CH}), 113.15(\mathrm{CH}), 69.29\left(\mathrm{CH}_{2}\right)$, $56.33\left(\mathrm{OCH}_{3}\right), 33.74\left(\mathrm{CH}_{2}\right)$. MS (ESI+), m/z: $481\left(\mathrm{M}^{2+}-\mathrm{H}^{+}\right)$. Anal. calcd. for $\mathrm{C}_{30} \mathrm{H}_{30} \mathrm{Br}_{2} \mathrm{~N}_{2} \mathrm{O}_{4}$ $(\mathrm{M}=642.38 \mathrm{~g} / \mathrm{mol}): \mathrm{C}, 56.09 ; \mathrm{H}, 4.71 ; \mathrm{N}, 4.36$. Found. C, 55.99; H, 4.86; N, 4.21.

1-[2-Oxo-2-(2-oxo-2H-chromen-3-yl)ethyl]-4-(2-\{1-[2-oxo-2-(2-oxo-2H-chromen-3-yl)ethyl]pyridin1-ium-4-yl\}ethyl)pyridin-1-ium dibromide (4c); White-beige crystals; yield $90 \% ; \mathrm{mp}>300{ }^{\circ} \mathrm{C}$. IR $\left(\mathrm{cm}^{-1}\right): 3,049,3,022,2,994,2,945,2,875,2,359,2,337,1,698,1,643,1,601,1,556,1,444,1,368$, 1,344, 1,184, 979. ${ }^{1} \mathrm{H}-\mathrm{NMR}$ (DMSO-d ${ }_{6}$ ) $\delta / \mathrm{ppm}$ : 8.96 (s, 2H, chromene), 8.89 (d, $J=6.4 \mathrm{~Hz}, 4 \mathrm{H}$, $\mathrm{H}_{\text {ortho/N+}}$ ), 8.21 (d, $J=6.4 \mathrm{~Hz}, 4 \mathrm{H}, \mathrm{H}_{\text {meta/ }}+$ ), 8.07 (d, $J=7.4 \mathrm{~Hz}, 2 \mathrm{H}$, chromene), 7.85 (t, $J=8.4 \mathrm{~Hz}$, 2H, chromene), 7.57 (d, $J=8.4 \mathrm{~Hz}, 2 \mathrm{H}$, chromene), 7.49 (t, $J=7.4 \mathrm{~Hz}, 2 \mathrm{H}$, chromene), 6.27(s, 4H, $\left.\mathrm{CH}_{2} / \mathrm{N}^{+}\right), 3.47\left(\mathrm{~s}, 4 \mathrm{H}, \mathrm{CH}_{2}-\mathrm{CH}_{2}\right) .{ }^{13} \mathrm{C}-\mathrm{NMR}\left(\mathrm{DMSO}-d_{6}\right) \delta / \mathrm{ppm}: 188.09(\mathrm{C}=\mathrm{O}), 158.73(\mathrm{C}=\mathrm{O}), 154.84$ (C), $149.52(\mathrm{CH}), 145.63(\mathrm{C}), 145.50(\mathrm{CH}), 131.39(\mathrm{C}), 128.32(\mathrm{CH}), 127.28(\mathrm{C}), 126.24(\mathrm{CH}), 125.37$ $(\mathrm{CH}), 121.19(\mathrm{CH}), 113.42(\mathrm{CH}), 61.04\left(\mathrm{CH}_{2}\right), 33.78\left(\mathrm{CH}_{2}\right)$. MS (ESI+), m/z: $557\left(\mathrm{M}^{2+}-\mathrm{H}^{+}\right)$. Anal. calcd. for $\mathrm{C}_{34} \mathrm{H}_{26} \mathrm{Br}_{2} \mathrm{~N}_{2} \mathrm{O}_{6}(\mathrm{M}=718.38 \mathrm{~g} / \mathrm{mol})$ : C, 56.84; H, 3.65; N, 3.90. Found: C, 56.99; H, 3.75; N, 3.75. 
1-[2-(1-Benzofuran-2-yl)-2-oxoethyl]-4-(2-\{1-[2-(1-benzofuran-2-yl)-2-oxoethyl]pyridin-1-ium-4yl\}ethyl)pyridin-1-ium dibromide (4d); Yellow-beige crystals; yield 76\%; mp $>300{ }^{\circ} \mathrm{C}$ (decomposition). IR ( $\left.\mathrm{cm}^{-1}\right)$ : 3,010, 2,970, 2,893, 2,631, 1,687, 1,643, 1,612, 1,549, 1,518, 1,474, 1,347, 1,291, 1,155, 1,136, 1,019, 928. ${ }^{1} \mathrm{H}-\mathrm{NMR}$ (DMSO-d $\left.)\right) \delta / \mathrm{ppm}: 9.02$ (d, $J=6.8 \mathrm{~Hz}, 4 \mathrm{H}, \mathrm{H}_{\text {ortho/N+ }}$ ), 8.30 (d, $J=6.8 \mathrm{~Hz}$, $4 \mathrm{H}, \mathrm{H}_{\text {meta/ }}+$ ) 8.20 (s, 2H, benzofuran), $7.97(\mathrm{dd}, J=8.0,1.0 \mathrm{~Hz}, 2 \mathrm{H}$, benzofuran), 7.85 (dd, $J=8.4$, $1.0 \mathrm{~Hz}, 2 \mathrm{H}$, benzofuran), 7.64-7.69 (m, 2H, benzofuran), 7.49-7.46 (m, 2H, benzofuran), 6.43 (s, 4H, $\mathrm{CH}_{2} / \mathrm{N}^{+}$), $3.52\left(\mathrm{~s}, 4 \mathrm{H}, \mathrm{CH}_{2}-\mathrm{CH}_{2}\right) .{ }^{13} \mathrm{C}-\mathrm{NMR}$ (DMSO-d $\left.d_{6}\right) \delta / \mathrm{ppm}: 181.31(\mathrm{C}=\mathrm{O}), 161.34(\mathrm{C}), 155.17(\mathrm{C})$, $149.36(\mathrm{C}), 145.81(\mathrm{CH}), 129.46(\mathrm{CH}), 127.51(\mathrm{CH}), 126.46(\mathrm{CH}), 125.02(\mathrm{C}), 124.12(\mathrm{CH}), 115.69$ $(\mathrm{CH}), 112.37(\mathrm{CH}), 64.86\left(\mathrm{CH}_{2}\right), 33.75\left(\mathrm{CH}_{2}\right) . \mathrm{MS}(\mathrm{ESI}+), m / z: 501\left(\mathrm{M}^{2+}-\mathrm{H}^{+}\right)$. Anal. calcd. for $\mathrm{C}_{32} \mathrm{H}_{26} \mathrm{Br}_{2} \mathrm{~N}_{2} \mathrm{O}_{4}(\mathrm{M}=662.37 \mathrm{~g} / \mathrm{mol}): \mathrm{C}, 58.03 ; \mathrm{H}, 3.96 ; \mathrm{N}, 4.23$. Found: $\mathrm{C}, 58.18 ; \mathrm{H}, 4.14 ; \mathrm{N}, 4.08$.

1-\{2-[(4R)-4-Benzyl-2-oxo-1,3-oxazolidin-3-yl]-2-oxoethyl\}-4-[2-(1-\{2-[(4S)-4-benzyl-2-oxo-1,3oxazolidin-3-yl]-2-oxoethyl, pyridin-1-ium-4-yl)ethyl]pyridin-1-ium dichloride (4e); Beige-brown crystals; highly hygroscopic; yield 46\%; mp not determined. IR $\left(\mathrm{cm}^{-1}\right)$ : 3,375, 3,029, 2,361, 2,337, 1,747, 1,698, 1,639, 1,571, 1,512, 1,518, 1,472, 1,391, 1,276, 1,198, 1,116, 985. ${ }^{1} \mathrm{H}-\mathrm{NMR}$ (DMSO-d $\left.d_{6}\right) \delta / \mathrm{ppm}$ : $9.03\left(\mathrm{~d}, J=6.8 \mathrm{~Hz}, 4 \mathrm{H}, \mathrm{H}_{\text {ortho/N+ }}\right), 8.28$ (d, $\left.J=6.8 \mathrm{~Hz}, 4 \mathrm{H}, \mathrm{H}_{\text {meta/N+ }}\right), 7.38-7.27(\mathrm{~m}, 10 \mathrm{H}, \mathrm{Ph}), 6.14$ (s, 4H, $\mathrm{CH}_{2} / \mathrm{N}^{+}$), 4.72-4.66 (m, 2H, oxazolidine), 4.52-4.48 (m, 2H, oxazolidine), 4.37-4.34 (m, 2H, oxazolidine), 3.49 (s, 4H, $\mathrm{CH}_{2}-\mathrm{CH}_{2}$ ), 3.13-3.09 (m, 2H, $\mathrm{CH}_{2} /$ benzyl), 2.98-2.92 (m, 2H, $\mathrm{CH}_{2} /$ benzyl). ${ }^{13} \mathrm{C}-\mathrm{NMR}\left(\mathrm{DMSO}-d_{6}\right) \delta / \mathrm{ppm}: 165.74(\mathrm{C}=\mathrm{O}), 161.40(\mathrm{COO}), 153.42(\mathrm{C}), 145.92(\mathrm{CH}), 135.29(\mathrm{C})$, $129.54(\mathrm{CH}), 128.69(\mathrm{CH}), 127.31(\mathrm{CH}), 127.03(\mathrm{CH}), 67.56\left(\mathrm{CH}_{2}\right), 62.66\left(\mathrm{CH}_{2}\right), 54.61(\mathrm{CH}), 36.56$ $\left(\mathrm{CH}_{2}\right), 33.75\left(\mathrm{CH}_{2}\right)$. MS (ESI+), m/z: $619\left(\mathrm{M}^{2+}-\mathrm{H}^{+}\right)$. Anal. calcd. for $\mathrm{C}_{36} \mathrm{H}_{36} \mathrm{Cl}_{2} \mathrm{~N}_{4} \mathrm{O}_{6}(\mathrm{M}=691.60 \mathrm{~g} / \mathrm{mol})$ : C, 62.52; H, 5.25; N, 8.10. Found: C, 62.37; H, 5.55; N, 7.90.

1-[2-(2-Nitrophenyl)-2-oxoethyl]-4-\{1-[2-(2-nitrophenyl)-2-oxoethyl]pyridin-1-ium-4-yl\}pyridin-1-ium dibromide (5a); Red crystals; yield 96\%; mp 258-259 ${ }^{\circ} \mathrm{C}$ (decomposition). IR ( $\mathrm{cm}^{-1}$ ): 3,046, 2,830, 2,773, 1,716, 1,639, 1,525, 1,505, 1,449, 1,360, 1,338, 1,223, 1,203, 997. ${ }^{1} \mathrm{H}-\mathrm{NMR}$ (DMSO- $d_{6}$ )

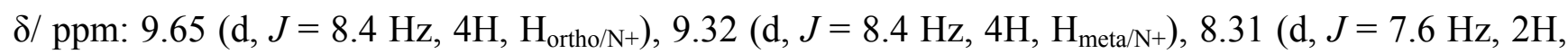
$\mathrm{Ph}), 8.12-8.07(\mathrm{~m}, 4 \mathrm{H}, \mathrm{Ph}), 7.98-7.94(\mathrm{~m}, 2 \mathrm{H}, \mathrm{Ph}), 6.47\left(\mathrm{~s}, 4 \mathrm{H}, \mathrm{CH}_{2} / \mathrm{N}^{+}\right) .{ }^{13} \mathrm{C}-\mathrm{NMR}\left(\mathrm{DMSO}-d_{6}\right) \delta$ ppm: $191.83(\mathrm{C}=\mathrm{O}), 158.75(\mathrm{C}), 157.59(\mathrm{CH}), 146.95(\mathrm{C}), 146.09(\mathrm{CH}), 134.55(\mathrm{CH}), 130.92(\mathrm{C})$, $128.92(\mathrm{CH}), 127.00(\mathrm{CH}), 124.63(\mathrm{CH}), 65.98\left(\mathrm{CH}_{2}\right)$. MS (ESI+), $m / z: 483\left(\mathrm{M}^{2+}-\mathrm{H}^{+}\right)$. Anal. calcd. for $\mathrm{C}_{26} \mathrm{H}_{20} \mathrm{Br}_{2} \mathrm{~N}_{4} \mathrm{O}_{6}(\mathrm{M}=644.27 \mathrm{~g} / \mathrm{mol})$ : C, 48.47; H, 3.13; N, 8.70. Found. C, 48.29; H, 3.28; N, 8.56.

1-[2-(2-Methoxyphenyl)-2-oxoethyl]-4-\{1-[2-(2- methoxyphenyl)-2-oxoethyl]pyridin-1-ium-4-yl\}pyridin1-ium dibromide (5b); Yellow crystals; yield 94\%; mp 252-253 ${ }^{\circ} \mathrm{C}$ (decomposition). IR ( $\left.\mathrm{cm}^{-1}\right)$ : 3,036, 2,988, 1,677, 1,641, 1,596, 1,485, 1,460, 1,434, 1,329, 1,280, 1,245, 1,197, 1,111, 1021, 987. ${ }^{1} \mathrm{H}-\mathrm{NMR}$ (DMSO- $\left.d_{6}\right) \delta /$ ppm: $9.35\left(\mathrm{~d}, J=7.2 \mathrm{~Hz}, 4 \mathrm{H}, \mathrm{H}_{\mathrm{ortho} / \mathrm{N}+}\right), 8.96\left(\mathrm{~d}, J=7.2 \mathrm{~Hz}, 4 \mathrm{H}, \mathrm{H}_{\text {meta/N+}}\right), 7.94(\mathrm{dd}$, $J=7.9,1.9 \mathrm{~Hz}, 2 \mathrm{H}, \mathrm{Ph}), 7.79$ (ddd, $J=8.5,7.2,1.9 \mathrm{~Hz}, 2 \mathrm{H}, \mathrm{Ph}), 7.41$ (dd, $J=8.5,1.0 \mathrm{~Hz}, 2 \mathrm{H}, \mathrm{Ph}$ ), $7.20(\mathrm{ddd}, 2 \mathrm{H}, J=7.9,7.2,1.0 \mathrm{~Hz}, 2 \mathrm{H}, \mathrm{Ph}), 6.38\left(\mathrm{~s}, 4 \mathrm{H}, \mathrm{CH}_{2} / \mathrm{N}^{+}\right), 4.10\left(\mathrm{~s}, 6 \mathrm{H}, \mathrm{OCH}_{3}\right) .{ }^{13} \mathrm{C}-\mathrm{NMR}$ $\left(\mathrm{DMSO}-d_{6}\right) \delta$ ppm: $190.89(\mathrm{C}=\mathrm{O}), 160.15(\mathrm{C}), 149.22(\mathrm{CH}), 147.21(\mathrm{CH}), 136.59(\mathrm{C}), 130.49(\mathrm{CH})$, $126.29(\mathrm{CH}), 122.80(\mathrm{C}), 121.03(\mathrm{CH}), 113.22(\mathrm{CH}), 69.98\left(\mathrm{CH}_{2}\right), 56.41\left(\mathrm{OCH}_{3}\right) . \mathrm{MS}(\mathrm{ESI}+), m / z: 453$ $\left(\mathrm{M}^{2+}-\mathrm{H}^{+}\right)$. Anal. calcd. for $\mathrm{C}_{28} \mathrm{H}_{26} \mathrm{Br}_{2} \mathrm{~N}_{2} \mathrm{O}_{4}(\mathrm{M}=614.33 \mathrm{~g} / \mathrm{mol}): \mathrm{C}, 54.74 ; \mathrm{H}, 4.27 ; \mathrm{N}, 4.56$. Found. C, 54.88; H, 4.41; N, 4.37. 
1-[2-Oxo-2-(2-oxo-2H-chromen-3-yl)ethyl]-4-\{1-[2-oxo-2-(2-oxo-2H-chromen-3-yl)ethyl]pyridin-1ium-4-ylspyridin-1-ium dibromide (5c); Yellow crystals; yield 93\%; mp $>300{ }^{\circ} \mathrm{C}$ (decomposition). IR ( $\left.\mathrm{cm}^{-1}\right): 3,052,2,990,2,931,1,695,1645,1,601,1,556,1,506,1,444,1,371,1,213,1,186,979$. ${ }^{1} \mathrm{H}-\mathrm{NMR}$ (DMSO- $d_{6}$ ) $\delta / \mathrm{ppm}: 9.27\left(\mathrm{~d}, J=6.6 \mathrm{~Hz}, 4 \mathrm{H}, \mathrm{H}_{\text {ortho/N+}}\right.$ ), 9.04 (s, 2H, chromene), 8.93 (d, $J=6.6 \mathrm{~Hz}, 4 \mathrm{H}, \mathrm{H}_{\text {meta }}+$ ) $), 8.16-8.10(\mathrm{~m}, 2 \mathrm{H}$, chromene), 7.96-7.85 (m, 2H, chromene), $7.83(\mathrm{~d}, J=8.4 \mathrm{~Hz}$, 2H, chromene), 7.59-7.49 (m, 2H, chromene), $6.44\left(\mathrm{~s}, 4 \mathrm{H}, \mathrm{CH}_{2} / \mathrm{N}^{+}\right) .{ }^{13} \mathrm{C}-\mathrm{NMR}\left(\mathrm{DMSO}-d_{6}\right) \delta / \mathrm{ppm}$ : $189.05(\mathrm{C}=\mathrm{O}), 160.53(\mathrm{C}=\mathrm{O}), 154.14(\mathrm{C}), 149.05(\mathrm{C}), 146.50(\mathrm{CH}), 141.65(\mathrm{CH}), 131.39(\mathrm{CH}), 128.45$ $(\mathrm{CH}), 126.28(\mathrm{CH}), 125.24(\mathrm{CH}), 122.37(\mathrm{C}), 120.19(\mathrm{C}), 115.42(\mathrm{CH}), 63.04\left(\mathrm{CH}_{2}\right) . \mathrm{MS}(\mathrm{ESI}+), m / z$ : $529\left(\mathrm{M}^{2+}-\mathrm{H}^{+}\right)$. Anal. calcd. for $\mathrm{C}_{32} \mathrm{H}_{22} \mathrm{Br}_{2} \mathrm{~N}_{2} \mathrm{O}_{6}(\mathrm{M}=690.33 \mathrm{~g} / \mathrm{mol}): \mathrm{C}, 55.67 ; \mathrm{H}, 3.21 ; \mathrm{N}, 4.06$. Found: C, 55.52; H, 3.12; N, 3.89 .

1-[2-(1-Benzofuran-2-yl)-2-oxoethyl]-4-\{1-[2-(1-benzofuran-2-yl)-2-oxoethyl]pyridin-1-ium-4-yl\}pyridin1-ium dibromide (5d); Green crystals; yield 78\%; mp 301-302 ${ }^{\circ} \mathrm{C}$ (decomposition). IR ( $\mathrm{cm}^{-1}$ ): 3,117, 3,055, 2,990, 2,892, 1,684, 1,639, 1,613, 1,550, 1,506, 1,455, 1,357, 1,342, 1,290, 1,155, 1,136, 1,017,

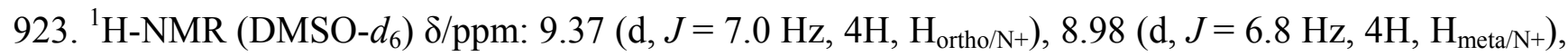
$8.25(\mathrm{~d}, J=1.0 \mathrm{~Hz}, 2 \mathrm{H}$, benzofuran), $8.01-7.99$ (m, 2H, benzofuran), 7.88 (dd, $J=8.4,0.9 \mathrm{~Hz}, 2 \mathrm{H}$, benzofuran), 7.69 (ddd, $J=8.4,7.2,1.3 \mathrm{~Hz}, 2 \mathrm{H}$, benzofuran), 7.50 (ddd, $J=8.1,7.2,0.9 \mathrm{~Hz}, 2 \mathrm{H}$, benzofuran), $6.46\left(\mathrm{~s}, 4 \mathrm{H}, \mathrm{CH}_{2} / \mathrm{N}^{+}\right) .{ }^{13} \mathrm{C}-\mathrm{NMR}$ (DMSO- $\left.d_{6}\right) \delta / \mathrm{ppm}: 181.31(\mathrm{C}=\mathrm{O}), 155.23(\mathrm{C}), 150.78$ (C), $149.70(\mathrm{C}), 147.34(\mathrm{CH}), 129.58(\mathrm{CH}), 126.61(\mathrm{CH}), 126.46(\mathrm{CH}), 125.25(\mathrm{C}), 124.17(\mathrm{CH})$, $115.88(\mathrm{CH}), 112.41(\mathrm{CH}), 65.56\left(\mathrm{CH}_{2}\right)$. MS (ESI+), $m / z: 473\left(\mathrm{M}^{2+}-\mathrm{H}^{+}\right)$. Anal. calcd. for $\mathrm{C}_{30} \mathrm{H}_{22} \mathrm{Br}_{2} \mathrm{~N}_{2} \mathrm{O}_{4}(\mathrm{M}=634.31 \mathrm{~g} / \mathrm{mol})$ : C, 56.80; H, 3.50; N, 4.42. Found: C, 57.01; H, 3.68; N, 4.32.

\subsection{Determination of Molecular Hydrophobicity}

The chromatographic $\mathrm{R}_{\mathrm{M}}$ value, related to the logarithm of the partition coefficient, can be used to estimate the molecular hydrophobicity of the bis-PyQAs [24]. The $\mathrm{R}_{\mathrm{M}}$ values were determined by thin layer partition chromatography, using acetonitrile/ethyl alcohol (10:10) at $25{ }^{\circ} \mathrm{C}$ for $30 \mathrm{~min}$. The $\mathrm{R}_{\mathrm{M}}$ value is defined as $R_{M}=\log \left(\left(1 / R_{f}\right)-1\right)$, where $R_{f}$ is the flow rate; $R_{f}=$ (solute velocity/mobile phase velocity).

\subsection{Antimicrobial Activity}

The inhibitory potential of the investigated compounds was tested against nine microorganisms, including Gram-positive bacteria (Bacillus subtilis, Bacillus cereus, Sarcina lutea), yeasts (Rhodotorula glutinis, Candida utilis, Saccharomyces cerevisiae) and molds (Aspergillus niger, Geotrichum candidum, Penicillium roqueforti). All of the bacterial and fungal strains were isolated from food spoilage microbiota and are all included in the collection of microorganisms (coded MIUG) of the Bioaliment Platform, Faculty of Food Science and Engineering, Dunarea de Jos University of Galati.

The cultures of indicator microorganisms were maintained in medium agar slants at $4{ }^{\circ} \mathrm{C}$ and used as stock cultures. The microorganism cultures were prepared from the stock cultures, through cultivation for $24 \mathrm{~h}$ at $37^{\circ} \mathrm{C}$ on nutrient agar, for bacteria, and for $72 \mathrm{~h}$ at $25^{\circ} \mathrm{C}$ on Sabouraud agar, for fungi.

The agar diffusion method was used for the qualitatively assessment of the antibacterial and antifungal properties of the synthesized compounds. Samples $(1 \mathrm{~mL})$ of each test microorganism 
suspension $\left(10^{6}\right.$ colony-forming units $\mathrm{CFU} / \mathrm{mL}$ for bacteria and yeasts and of $10^{5}-10^{6}$ spores $/ \mathrm{mL}$ for molds) obtained from microorganism cultures by serial dilutions were inoculated at $42{ }^{\circ} \mathrm{C}$ in sterile agar culture medium, in sterile Petri dishes. After homogenization and solidification of the medium, sterile $19 \mathrm{~mm}$-diameter paper discs, loaded with $30 \mu \mathrm{L}$ from $5 \mathrm{mg} / \mathrm{mL}$ aqueous solutions of each tested bis-PyQA were aseptically placed on the surface. Sterile water-impregnated discs were used as blanks. The plates inoculated with bacteria were incubated at $37{ }^{\circ} \mathrm{C}$ for $48 \mathrm{~h}$ and those with fungi at $25^{\circ} \mathrm{C}$ for 3-5 days. After $24 \mathrm{~h}$ of cultivation, the diameters of the inhibition zones (Diz, mm) were measured to the nearest $0.5 \mathrm{~mm}$, compared to the blank, under the same standardized conditions. The evaluation of microbiostatic or microbicide effects of the studied chemical compounds was also made by checking some morphological characteristics of colonies, i.e., pigmentation and sporulation intensity.

The quantitative effect of the substances with the higher inhibitory potential of cells growth was determined in vitro, by microbial cultivation in stationary conditions, in liquid nutrient broth medium, on the basis of the minimum inhibitory concentration (MIC) values. The most sensitive microorganisms were used for quantitative evaluation. Serial dilutions $(5,2.5,1.25,0.625,0.3125$, $0.1562,0.0781,0.0390,0.0190$ and $0.0097 \mathrm{mg}$ of chemical compound $/ \mathrm{mL}$ medium) were prepared from stock solutions $(5 \mathrm{mg} / \mathrm{mL}) ; 100 \mu \mathrm{L}$ of each standardized microbial suspension was added to an equal volume of each chemical compound dilution (excluding the sterility control). After incubation for $24 \mathrm{~h} \pm 1 \mathrm{~h}$ at $37^{\circ} \mathrm{C}$ for bacteria, the turbidity of the cultures associated with the veil formation at the medium's surface or sediment formation was visually assessed. The microorganism's growth was monitored by optical density determination $\left(\mathrm{OD}_{620 \mathrm{~nm}}\right)$ using the microplate reader. The tests were performed simultaneously on negative controls (only medium), positive controls (medium with test microorganism) and sterility controls (medium with chemical compounds). The lowest concentration of antimicrobial agent that inhibits the development of visible growth was taken as the minimum inhibitory concentration. All of the experiments were performed in triplicate.

\section{Conclusions}

We report here the efficient synthesis of nine new symmetrical diquaternary salts by alkylation of either 4-[2-(pyridin-4-yl)ethyl]pyridine or 4,4'-bipyridine, with various bromo- or chloro-acetophenone analogues. It appeared that the reaction was more efficient with 4,4'-bipyridine compared to 4-[2-(pyridin-4-yl)ethyl]pyridine. The antimicrobial properties for eight salts were investigated through disk diffusion assay, revealing that all compounds display low to high activity against the tested microorganisms and a broad spectrum of activity. Compounds $4 \mathbf{a}-\mathbf{d}$, 5a and $\mathbf{5 d}$ show efficient inhibitory properties at least against one bacterial strain. However, the most active bis-PyQA was Compound $\mathbf{5 b}$, which showed a broad spectrum of activity. We found some indications that the antibacterial properties seem to correlate well with $\operatorname{low} \log P$ and van der Waals surface area values.

All of the new compounds showed significant in vitro antibacterial activity, the Compounds $\mathbf{4 b}$ and $\mathbf{5 b}$ having superior antimicrobial activity against all microorganisms under investigation. Further studies should be done to elucidate their mechanism of action and to determine whether their activity is lethal, or merely inhibitory to microorganisms. Preliminary studies [31] showed that some of these salts are also able to prevent or destroy biofilm formation with microorganisms from food industry hygiene. 
Our results suggest that we have uncovered new leads that may proceed further in the development of antibacterial agents.

\section{Supplementary Materials}

Supplementary materials can be accessed at: http://www.mdpi.com/1420-3049/19/8/11572/s1.

\section{Acknowledgments}

This work was supported by a grant of the Romanian National Authority for Scientific Research, CNCS-UEFISCDI, Project Number PN-II-ID-PCE-2011-3-0226. This work has been also partially supported by the Labex ARCANE (ANR-11-LABX-0003-01).

\section{Author Contributions}

All authors contributed equally to this work.

\section{Conflicts of Interest}

The authors declare no conflict of interest.

\section{References}

1. Kim, Y.H.; Sun, G. Functional finishing of acrylic and cationic dyeable fabrics: Intermolecular interactions. Text. Res. J. 2002, 72, 1052-056.

2. Cai, Z.; Sun, G. Antimicrobial finishing of acrilan fabrics with cetylpyridinium chloride. J. Appl. Polym. Sci. 2004, 94, 243-247.

3. Son, Y.A.; Sun, G. Durable antimicrobial Nylon 66 fabrics: Ionic interactions with quaternary ammonium salts. J. Appl. Polym. Sci. 2003, 90, 2194-2199.

4. Ishikawa, S.; Matsumura, Y.; Yoshizako, F.; Tsuchido, T. Characterization of a cationic surfactant-resistant mutant isolated spontaneously from Escherichia coli. J. Appl. Microbiol. 2002, 92, 261-268.

5. Seong, H.; Whang, H.S.; Ko, S. Synthesis of a quaternary ammonium derivative of chito-oligosaccharide as antimicrobial agent for cellulosic fibers. J. Appl. Polym. Sci. 2000, 76, 2009-2015.

6. Augusta, S.; Gruber, H.F.; Streichsbier, F. Synthesis and antibacterial activity of immobilized quaternary ammonium salts. J. Appl. Polym. Sci. 1994, 53, 1149-1163.

7. Isquith, A.; Abbot, A.; Walters, P.A. Surface-bonded antimicrobial activity of an organosilicon quaternary ammonium chloride. Appl. Environ. Microbiol. 1972, 24, 859-863.

8. Ancelin, M.L.; Calas, M.; Bonhoure, A.; Herbute, S.; Vial, H.J. In vivo antimalarial activities of mono-and bis quaternary ammonium salts interfering with Plasmodium phospholipid metabolism. Antimicrob. Agents Ch. 2003, 47, 2598-2605. 
9. Chanawanno, K.; Chantrapromma, S.; Anantapong, T.; Kanjana-Opas, A.; Fun, H.-K. Synthesis, structure and in vitro antibacterial activities of new hybrid disinfectants quaternary ammonium compounds: Pyridinium and quinolinium stilbene benzenesulfonates. Eur. J. Med. Chem. 2010, 45, 4199-4208.

10. Mammeri, H.; Nordmann, P. Extended-spectrum cephalosporinases in Enterobacteriaceae. Anti-Infect. Agents Med. Chem. 2007, 6, 71-82.

11. Obando, D.; Pantarat, N.; Handke, R.; Koda, Y.; Widmer, F.; Djordjevic, J.T.; Ellis, D.H.; Sorrell, T.C.; Jolliffe, K.A. Synthesis, antifungal, haemolytic and cytotoxic activities of a series of bis(alkylpyridinium)alkanes. Bioorgan. Med. Chem. 2009, 17, 6329-6339.

12. Kourai, H.; Yabuhara, T.; Shirai, A.; Maeda, T.; Nagamune, H. Synthesis and antimicrobial activities of a series of new bis-quaternary ammonium compounds. Eur. J. Med. Chem. 2006, 41, 437-444.

13. Lu, D.N.; Zhou, X.R.; Xing, X.D.; Wang, X.G.; Liu, Z. Quaternary ammonium salts (QAS) grafted cellulose fiber: Preparation and antimicrobial function. Acta Polym. Sin. 2004, 14, 107-113.

14. Lucchese, A.M.; Marzorati, L. Catálise de transferência de fase. Quim. Nova 2000, 23, 641-652.

15. Tehfe, M.-A.; Zein-Fakiha, A.; Lalevée, J.; Dumur, F.; Gigmes, D.; Graff, B.; Morlet-Savarya, F.; Hamieh, T.; Fouassier, J.-P. New pyridinium salts as versatile compounds for dye sensitized photopolymerization. Eur. Polym. J. 2013, 49, 567-574.

16. Pernak, J.; Rogoza, J. Synthesis of 3-substituted pyridinium salts. ARKIVOC 2000, 1, 889-904.

17. Pellissier, H. Asymmetric 1,3-dipolar cycloadditions. Tetrahedron 2007, 63, 3235-3285.

18. Causin, V.; Saielli, G. Effect of a structural modification of the bipyridinium core on the phase behaviour of, viologen-based bistriflimide salts. J. Mol. Liq. 2009, 145, 41-47.

19. Rowley, N.M.; Mortimer, R.J. New electrochromic materials. Sci. Progr. London 2002, 85, 243-262.

20. Pisarenko, S.V.; Demidov, O.P.; Aksenov, A.V.; Borovlev, I.V. Synthesis and hydroxylation of 1-alkyl- and 7-alkyl- 1,3,7-triazapyrenium salts. Chem. Heterocycl. Comp. 2009, 45, 580-586.

21. Furdui, B.; Dinica, R.M.; Tabacaru, A.; Pettinari, C. Synthesis and physico-chemical properties of a novel series of aromatic electron acceptors based on N-heterocycles. Tetrahedron 2012, 68, 6164-6168.

22. Dinica, R.; Furdui, B.; Bahrim, G.; Demeunynck, M. Precurseurs de nouveaux heterocycles d'intéret biologique. Rev. Roum. Chim. 2008, 53, 21-24.

23. Shuchismita, D.; Adhikary, K.K.; Kim, C.K.; Lee, B.-S.; Lee, H.W. Nucleophilic substitution reactions of $\alpha$-chloroacetanilides with pyridines in dimethyl sulfoxide. Bull. Korean Chem. Soc. 2005, 26, 776-780.

24. Furdui, B.; Bahrim, G; Dinica, R.; Druţă, I.; Demeunynck, M. In vitro antimicrobial activity of new nitrogen heterocycles derivates from 4,4'-bipyridine. Rom. Biotech. Lett. 2007, 12, 3073-3078.

25. Furdui, B.; Constantin, O.; Tabacaru, A.; Dinica, R. New bis-pyridinium diquaternary salts with antimicrobial properties. Rev. Chim. 2012, 63, 667-671.

26. Piddoc, L.-J.V. Techniques used for the determination of antimicrobial resistance and sensitivity in bacteria. J. Appl. Bactriol. 1990, 68, 307-318. 
27. Englera, A.C.; Wiradharmab, N.; Ongb, Z.Y.; Coadya, D.J.; Hedricka, J.L.; Yang, Y.-Y. Emerging trends in macromolecular antimicrobials to fight multi-drug-resistant infections. Nano Today 2012, 7, 201-222.

28. Huang, Y.; Wang, X.; Wan, H. Studies on mechanism of action of anticancer peptides by modulation of hydrophobicity within a defined structural framework. Mol. Cancer Ther. 2011, 10, 416-426.

29. Franke, R. Theoretical Drug Design Method; Elsevier Science Publishers: New York, NY, USA, 1984; pp. 30-79.

30. Calculator Plugins were used for structure property prediction and calculation, Marvin 6.0.2, 2013, ChemAxon. Available onlin: http://www.chemaxon.com accessed (accessed on 13 June 2014).

31. Furdui, B.; Constantin, O.; Dinica, R.; Bahrim, G. University "Dunarea de Jos" of Galaţi, Galaţi, Romania. Unpublished work, 2012.

Sample Availability: Not available.

(C) 2014 by the authors; licensee MDPI, Basel, Switzerland. This article is an open access article distributed under the terms and conditions of the Creative Commons Attribution license (http://creativecommons.org/licenses/by/3.0/). 